\title{
Molecular and Dissociative Adsorption of Oxygen on Au-Pd Bimetallic Clusters: Role of Composition and Spin State of the Cluster.
}

\author{
Manzoor Ahmad Dar* $*^{a}$ and Sailaja Krishnamurty ${ }^{b}$ \\ ${ }^{a}$ Department of Chemistry, Islamic University of Science and Technology, J\&K-192122, India \\ ${ }^{b}$ Physical Chemistry Division, CSIR-National Chemical Laboratory, Pune-411008, India
}

To whom correspondence should be addressed: manzoor.dar@islamicuniversity.edu.in

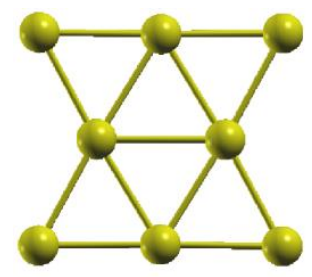

$\mathrm{Au}_{\mathbf{8}}$ (1.24)

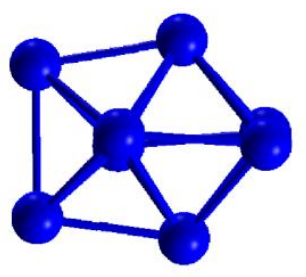

$\mathrm{Pd}_{8}$
$\mathrm{~S}=1$

$(0.13)$

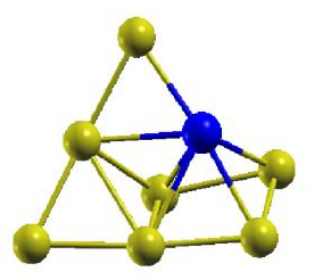

$\mathrm{Au}_{7} \mathbf{P d}_{1}$ $\mathrm{S}=4$
$(1.38$

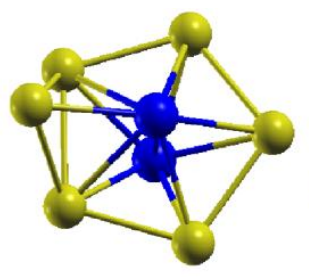

$\underset{\mathrm{S}=3}{\mathrm{Au}_{6} \mathrm{Pd}_{2}}$

(0.60)

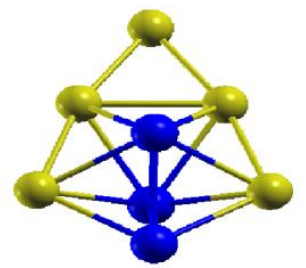

$\mathrm{Au}_{5} \mathrm{Pd}_{3}$ $\mathrm{S}=\mathbf{2}$ $(0.13)$

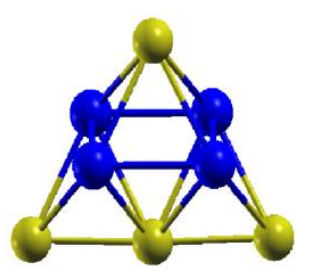

$\mathrm{Au}_{4} \mathrm{Pd}_{4}$

$\mathrm{S}=1$
$(0.19)$

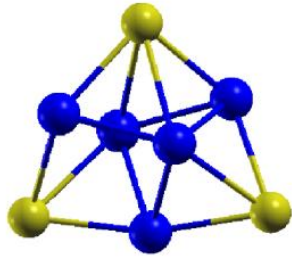

$\mathrm{Au}_{3} \mathrm{Pd}_{5}$

$\mathrm{S}=\mathbf{2}$

$(0.14)$

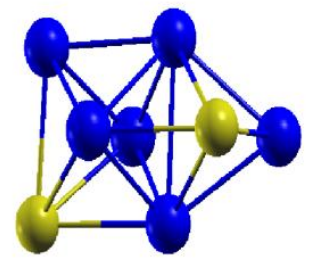

$\mathrm{Au}_{2} \mathrm{Pd}_{6}$

$S=1$
$(0.14)$

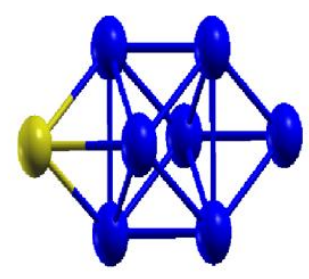

$\mathrm{Au}_{1} \mathrm{Pd}_{7}$

$\mathrm{S}=\mathbf{2}$

$(0.12)$

Figure S1: Optimized structures of the $\mathrm{Au}_{8}, \mathrm{Pd}_{8}$ and $\mathrm{Au}_{8-1} \mathrm{Pd}_{\mathrm{n}}$ with their relative energies in the highest energy spin states. 


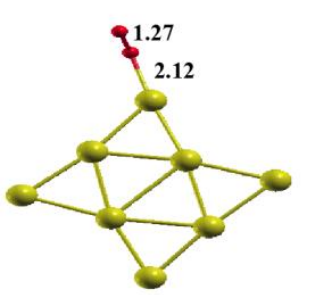

$\mathrm{Au}_{8} \mathrm{O}_{2}$ $\mathrm{S}=\mathbf{1}$

(1.06)

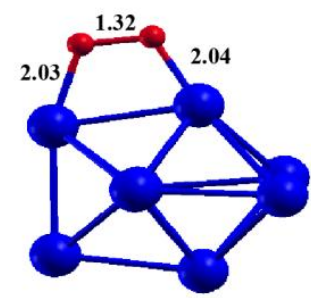

$\mathrm{Pd}_{8} \mathrm{O}_{2}$

$\mathrm{S}=1$

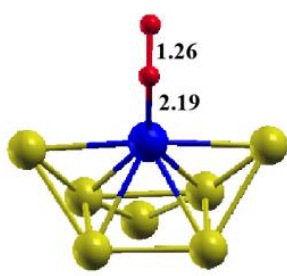

$\mathrm{Au}_{7} \mathrm{Pd}_{1} \mathrm{O}_{2}$

$\mathrm{S}=\mathbf{4}$

(0.30)

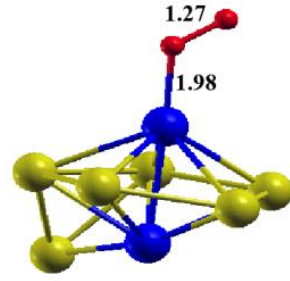

$\mathrm{Au}_{6} \mathrm{Pd}_{2} \mathrm{O}_{2}$

$\mathrm{S}=1$

$(0.53)$

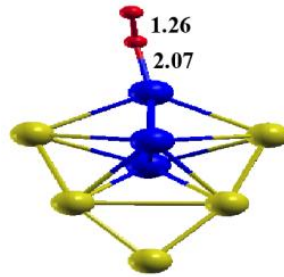

$\mathrm{Au}_{5} \mathrm{Pd}_{3} \mathrm{O}_{2}$ $\mathrm{S}=4$

$(0.12)$

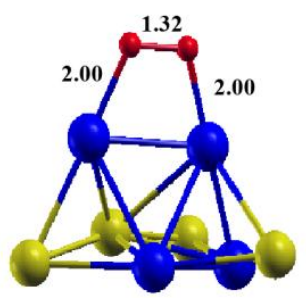

$\underset{\mathrm{S}=1}{\mathrm{Au}_{4} \mathrm{Pd}_{4} \mathrm{O}_{2}}$

(0.17)

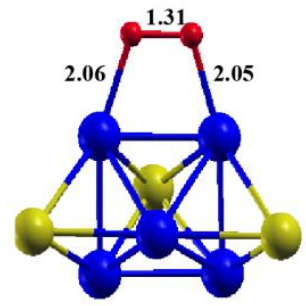

$\mathrm{Au}_{3} \mathrm{Pd}_{5} \mathrm{O}_{2}$

$\mathrm{S}=\mathbf{2}$

(0.02)

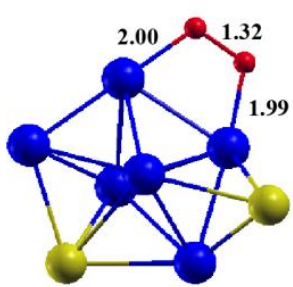

$\mathrm{Au}_{2} \mathrm{Pd}_{6} \mathrm{O}_{2}$

$\mathrm{S}=1$
$(0.00)$

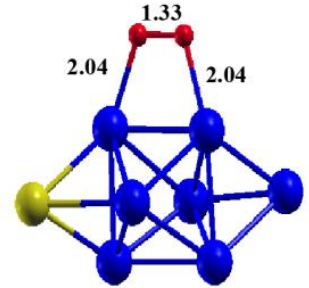

$\mathrm{Au}_{1} \mathbf{P d}_{7} \mathrm{O}_{2}$

$\mathrm{S}=4$

(0.38)

Figure S2: Optimized structures of the $\mathrm{O}_{2}$ adsorbed complexes of $\mathrm{Au}_{8}, \mathrm{Pd}_{8}$ and $\mathrm{Au}_{8-1} \mathrm{Pd}_{\mathrm{n}}$ in their high energy spin states with their relative energies.
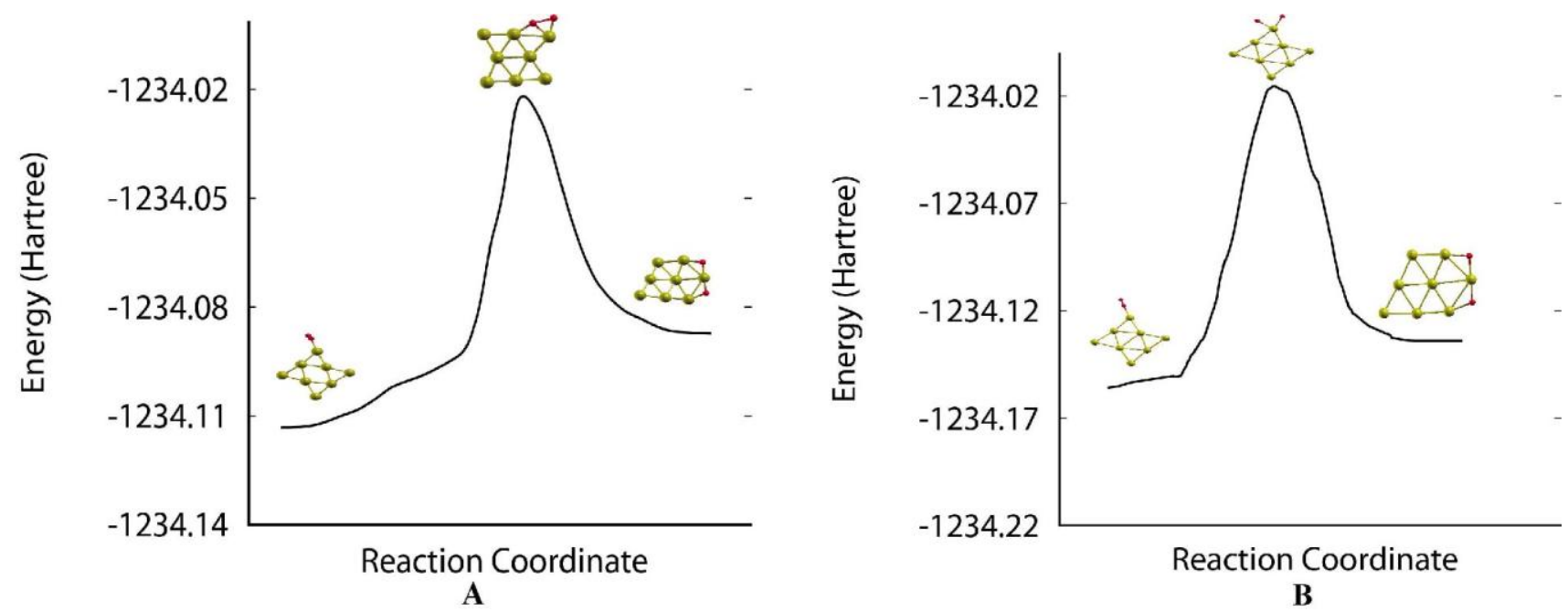

Figure S3: Internal reaction coordinate (IRC) calculation for $\mathrm{O}_{2}$ dissociation on $\mathrm{Au}_{8}$ cluster along (A) singlet and (B) triplet reactions channels. 